\title{
Decreased mitochondrial priming determines chemoresistance of colon cancer stem cells
}

\author{
S Colak ${ }^{1}$, CD Zimberlin ${ }^{1}$, E Fessler ${ }^{1}$, L Hogdal ${ }^{2}$, PR Prasetyanti ${ }^{1}$, CM Grandela ${ }^{1,3}$, A Letai ${ }^{2}$ and JP Medema*,1
}

Tumor heterogeneity is in part determined by the existence of cancer stem cells (CSCs) and more differentiated tumor cells. CSCs are considered to be the tumorigenic root of cancers and suggested to be chemotherapy resistant. Here we exploited an assay that allowed us to measure chemotherapy-induced cell death in CSCs and differentiated tumor cells simultaneously. This confirmed that CSCs are selectively resistant to conventional chemotherapy, which we revealed is determined by decreased mitochondrial priming. In agreement, lowering the anti-apoptotic threshold using ABT-737 and WEHI-539 was sufficient to enhance chemotherapy efficacy, whereas ABT-199 failed to sensitize CSCs. Our data therefore point to a crucial role of BCLXL in protecting CSCs from chemotherapy and suggest that BH3 mimetics, in combination with chemotherapy, can be an efficient way to target chemotherapy-resistant CSCs.

Cell Death and Differentiation (2014) 21, 1170-1177; doi:10.1038/cdd.2014.37; published online 28 March 2014

Colorectal cancer is the third most common cancer worldwide. ${ }^{1,2}$ Patients with advanced stage colorectal cancer are routinely treated with 5-fluorouracil (5-FU), leucovorin and oxaliplatin (FOLFOX), or with 5-FU, leucovorin and irinotecan (FOLFIRI), often in combination with targeted agents such as anti-VEGF or anti-EGFR at metastatic disease. ${ }^{3-6}$ Despite this intensive treatment, therapy is still insufficiently effective and chemotherapy resistance occurs frequently. Although still speculative, it has been suggested that unequal sensitivity to chemotherapy is due to an intratumoral heterogeneity that is orchestrated by cancer stem cells (CSCs) that can self-renew and give rise to more differentiated progeny. ${ }^{7,8}$ When isolated from patients, CSCs efficiently form tumors upon xenotransplantation into mice which resemble the primary tumor from which they originated. ${ }^{9-11}$ In addition, many xenotransplantation studies have emphasized the importance of CSCs for tumor growth. ${ }^{9-12}$ Colon CSCs were originally isolated from primary human colorectal tumor specimens using CD133 as cell surface marker and shown to be highly tumorigenic when compared with the non-CSCs population within a tumor. 9,10 Later, other cell surface markers as well as the activity of the Wnt pathway have been used to isolate colon CSCs from tumors. $^{12,13}$ Spheroid cultures have been established from human primary colorectal tumors that selectively enrich for the growth of colon CSCs, ${ }^{11,12}$ although it is important to realize that these spheres also contain more differentiated tumor cells. ${ }^{12}$ In agreement, we have shown that the Wnt activity reporter that directs the expression of enhanced green fluorescent protein (TOP-GFP) allows for the separation of CSCs from more differentiated progeny in the spheroid cultures. $^{12}$
CSCs are suggested to be responsible for tumor recurrence after initial therapy, as they are considered to be selectively resistant to therapy. ${ }^{11,14}$ Conventional chemotherapy induces, among others, DNA damage and subsequent activation of the mitochondrial cell death pathway, which is regulated by a balance between pro- and anti-apoptotic BCL2 family members. ${ }^{15}$ Upon activation of apoptosis, pro-apoptotic $\mathrm{BH} 3$ molecules are activated and these may perturb the balance in favor of apoptosis initiated by mitochondrial outer membrane polarization (MOMP), release of cytochrome $c$ and subsequent activation of a caspase cascade.

The apoptotic balance of cancer cells can be measured with the use of a functional assay called $\mathrm{BH} 3$ profiling. ${ }^{16} \mathrm{BH} 3$ profiling is a method to determine the apoptotic 'priming' level of a cell by exposing mitochondria to standardized amounts of roughly 20-mer peptides derived from the alpha-helical $\mathrm{BH} 3$ domains of $\mathrm{BH} 3-o n l y$ proteins and determining the rate of mitochondrial depolarization. Using this approach, priming was measured in various cancers and compared with normal tissues. ${ }^{17,18}$ In all cancer types tested, the mitochondrial priming correlated well with the observed clinical response to chemotherapy. That is, cancers that are highly primed are more chemosensitive, whereas chemoresistant tumor cells and normal tissues are poorly primed. ${ }^{17,18}$ This suggests that increasing mitochondrial priming can potentially increase chemosensitivity, which can be achieved by directly inhibiting the anti-apoptotic BCL2 family members. ${ }^{18}$ To this end, smallmolecule inhibitors, so-called $\mathrm{BH} 3$ mimetics, have been developed. ABT-737 and the highly related $A B T-263$ both inhibit BCL2, BCLXL and BCLW ${ }^{19-21}$ and were shown to be effective in killing cancer cells in vitro and in vivo ${ }^{21}$ with a

${ }^{1}$ LEXOR (Laboratory of Experimental Oncology and Radiobiology), Center for Experimental Molecular Medicine, Academic Medical Center, University of Amsterdam, Amsterdam, The Netherlands and ${ }^{2}$ Department of Medical Oncology, Dana-Farber Cancer Institute, 450 Brookline Avenue, Boston, MA, USA

${ }^{*}$ Corresponding author: JP Medema, LEXOR (Laboratory for Experimental Oncology and Radiobiology), Center for Experimental Molecular Medicine, Academic Medical Center, University of Amsterdam, CEMM Room G2-131, AMC, Meibergdreef 9, Amsterdam 1105, AZ, The Netherlands. Tel: +31 205667777 ; Fax: +31 206977192 ; E-mail: J.P.Medema@amc.uva.nl

${ }^{3}$ Current address: Pluriomics BV, Leiden 2333BD, The Netherlands

Abbreviations: CSC, cancer stem cell; MOMP, mitochondrial outer membrane permeabilization; BH3, BCL2 homology domain 3; TOP-GFP, Tcf/Lef optimal promotergreen fluorescent protein; 5-FU, 5-fluorouracil; FOLFOX, 5-FU, leucovorin and oxaliplatin; FOLFIRI, 5-FU, leucovorin and irinotecan

Received 07.8.13; revised 09.1.14; accepted 09.2.14; Edited by G Melino; published online 28.3.14 
preference for BCL2. ${ }^{19,22}$ As BCL2 protein expression is often upregulated in hematopoietic cancers, it represents a promising target, which was supported by high efficacy of these $\mathrm{BH} 3$ mimetics in animal experiments. ${ }^{21}$ However, in vivo efficacy is limited due to thrombocytopenia, which relates to a dependence of platelets on $B C L X L$ for survival. $^{23,24}$ To overcome this toxicity, a BCL2-specific compound, ABT-199, was developed. ${ }^{25}$ Souers et al. ${ }^{25}$ showed that inhibition of BCL2 using ABT-199 blocks tumor growth of acute lymphoblastic leukemia cells in xenografts. In addition to the single compound effects of ABT-199, combination with rituximab inhibited growth of non-Hodgkin's lymphoma, mantle cell lymphoma and acute lymphoblastic leukemia tumor cells growth in vivo. ${ }^{25}$ Moreover, highly effective tumor lysis was observed in all three patients with chronic lymphocytic leukemia that were treated with ABT199. ${ }^{25}$ More recently, a BCLXL-specific compound, WEHI-539, was discovered using high-throughput chemical screening. ${ }^{26}$

As the apoptotic balance appears a useful target for the treatment of cancers and CSCs have been suggested to resist therapy selectively, we set out to analyze whether specifically colon CSCs are resistant to therapy and whether this is due to an enhanced anti-apoptotic threshold, specific to CSCs. To study chemosensitivity, we developed a robust single cell-based analysis in which we can measure apoptosis simultaneously in CSCs and their differentiated progeny. Utilizing this system we showed that colon CSCs and not their differentiated progeny are resistant to chemotherapeutic compounds and that this was due to the fact that these cells are less primed to mitochondrial death. Furthermore, inhibition of anti-apoptotic BCLXL molecule with either ABT-737 or WEHI-539, but not ABT-199, breaks this resistance and sensitizes the CSCs to chemotherapy.

\section{Results}

Colon CSCs are resistant to various drugs. Previously, cell death in colon CSCs and differentiated tumor cells has been compared by utilizing spheroid cultures enriched for CSCs in comparison with such cultures that were forced to undergo differentiation in vitro. ${ }^{11,27}$ In this approach, differentiated tumor cells, also called sphere-derived adherent cultures (SDAC), were generated by placing spheroids in adherent cultures using medium containing fetal calf serum. Chemotherapy-induced cell death was subsequently compared between spheroids and SDAC cultures. This approach therefore compares mixtures of cells maintained under different conditions and observed difference may not necessarily relate to intrinsic resistance of CSCs. Here we developed a robust assay, which allows treatment and cell death measurement of colon CSCs and their differentiated progeny under the exact same conditions. As we have shown that Wnt activity discriminates between CSCs and differentiated progeny within one sphere, ${ }^{12}$ we used this segregation to measure cell death in spheroid cultures after chemotherapy treatment with CaspGlow, a FACS-based assay that measures caspase- 3 activity at the single-cell level (Figure 1a).

This assay revealed that CSCs were more resistant to conventional chemotherapeutic compounds, including oxaliplatin (Figures 1a and b), cisplatin, 5-FU, etoposide, the chemotherapy regimens FOLFIRI and FOLFOX and also to the death receptor ligand TRAIL (Figure 1b). In addition to caspase-3 activity, other apoptotic features were measured at the single-cell level using a similar FACS-based setup. Phosphatidylserine exposure (Figure 2a) as well as loss of mitochondrial potential (Figure $2 b$ ) confirmed that colon CSCs were more resistant to chemotherapy when compared with their differentiated progeny. To show that resistance of colon CSCs toward chemotherapy is a common phenomenon, various spheroid cultures derived from different patients with colorectal cancer (Co108 and Co123) were transduced with TOP-GFP. As shown before, ${ }^{12}$ also in these spheroid cultures, WNT reporter activity correlated with CD133 expression and clonogenic potential (Supplementary Figure 1), pointing to the presence of CSCs in the CD133 $3^{\text {high }}$ and TOP-GFPhigh fraction. Treatment of these distinct colorectal spheroid cultures with oxaliplatin also revealed a differential resistance to oxaliplatin, in which CSCs (TOP-GFP ${ }^{\text {high }}$ ) are more resistant when compared with differentiated tumor cells (TOP-GFP ${ }^{\text {low }}$ ) (Figure 2c).

Colon CSCs are less primed to death. Several distinct mechanisms have been described that can lead to resistance toward chemotherapy. One of these involves a block in apoptosis induction, which can be caused by a shift in the apoptotic balance, where a deregulation of anti- and pro-apoptotic signals is tilted in favor of anti-apoptotic proteins. ${ }^{28}$ In agreement, colon cancer spheroids overexpressing the anti-apoptotic BCL2 members BCL2, BCLW and BCLXL (Figure $3 a$ and Supplementary Figures 2a and c) were completely resistant to oxaliplatin when analyzed in the CaspGlow assay (Figure $3 \mathrm{~b}$ and Supplementary Figure 2c). Ectopic overexpression of the other BCL2 proteins BFL1, BCLB and MCL1 was not sufficient to overcome oxaliplatin-induced cell death in spheroid cultures (Supplementary Figure 2), suggesting that changing the apoptotic balance using the previously reported stable anti-apoptotic BCL2 family members ${ }^{19}$ is indeed a possible means to escape from chemotherapy.

To directly evaluate whether the resistance of CSCs is due to a difference in this apoptotic balance, $\mathrm{BH} 3$ profiling experiments were used to measure the level of mitochondrial priming in colon CSCs and differentiated tumor cells (Figures $3 c$ and d). Spheroid cultures were treated with $\mathrm{BH} 3$ peptides and mitochondrial depolarization was measured. Importantly, we have shown before that these $\mathrm{BH} 3$ peptides have differential binding to anti-apoptotic BCL2 family proteins. ${ }^{29}$ For instance, HRK peptide has a much greater affinity for BCLXL than BCL2 or BCLW (Figure 3c). For all BH3 peptides, less depolarization was measured in CSCs when compared with differentiated cells (Figure $3 d$ and Supplementary Figure 3 ). This profiling indicates that colon CSCs display decreased mitochondrial priming in comparison to differentiated tumor cells and thereby can resist chemotherapy.

BCLXL-specific inhibitor WEHI-539 sensitizes colon cancer CSCs toward chemotherapy. In some cancers, expression of anti-apoptotic molecules is highly enhanced 
a

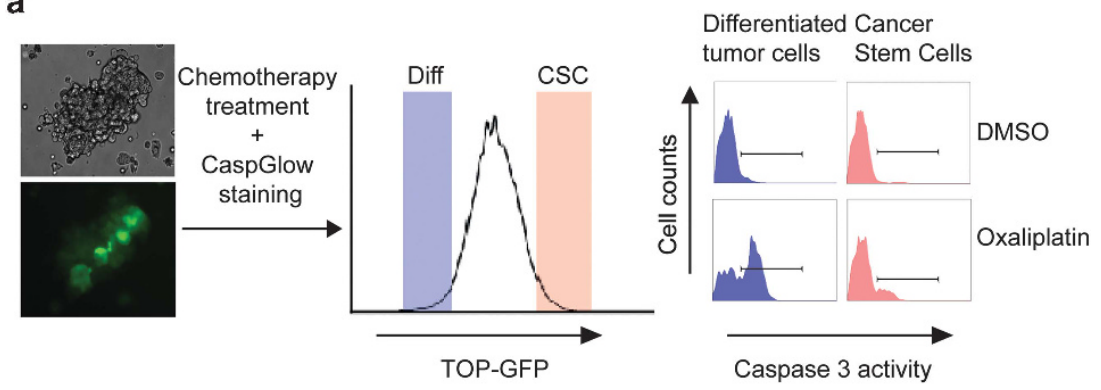

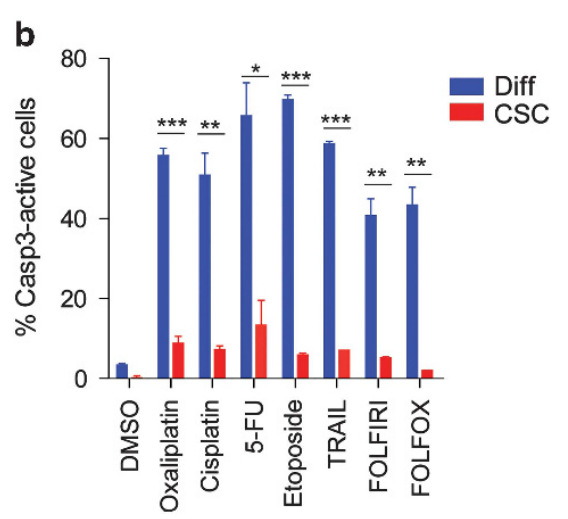

Figure 1 Colon cancer stem cells (CSCS) are resistant toward chemotherapy. (a) Spheroid primary cultures were derived from primary human colorectal tumors and transduced with TOP-GFP construct. In these cultures, Wnt pathway activity drives the expression of GFP (TOP-GFP). These spheroid cultures with TOP-GFP (Co100) were treated with chemotherapy, for example, oxaliplatin for $24 \mathrm{~h}$. Subsequently, spheroid cultures were stained with CaspGlow to measure caspase-3 activity. Cells are first gated on TOP-GFPlow (blue bar) and TOP-GFPhigh (red bar), differentiated tumor cells and CSCs, respectively. Caspase-3 activity is then separately analyzed for the differentiated tumor cells and CSCs. (b) This method was used to test various chemotherapeutic therapies. Spheroid cultures treated for $24 \mathrm{~h}$ with oxaliplatin, cisplatin, 5 -FU, etoposide, FOLFIRI and FOLFOX or $4 \mathrm{~h}$ with TRAIL are shown. Caspase-3 activity shows more cell death in differentiated cells compared with CSCs. Significance comparing differentiated versus CSCs is indicated $\left({ }^{\star} P<0.05,{ }^{\star \star} P<0.01,{ }^{* * \star} P<0.001\right)$

when compared with the untransformed cells from the same patient. ${ }^{30-32}$ As overexpression of BCL2, BCLXL or BCLW could enhance chemotherapy resistance (Supplementary Figure 2c), we analyzed whether the small-molecule inhibitor ABT-737, which binds to all these anti-apoptotic family members, ${ }^{21}$ could target CSCs. In addition, we utilized the more selective compounds ABT-199, which solely targets BCL2, and WEHI-539, which solely targets BCLXL. First we tested whether the mitochondrial pathway regulates clonogenic potential of CSCs. Limiting dilution analysis with CSCs that were pre-treated with ABT-737, ABT-199 or WEHI-539 revealed that $A B T-737$ and WEHI-539 both were sufficient to decrease clonogenic capacity, whereas ABT-199 did not affect clonogenic growth (Figure 4a). As WEHI-539 is selective for BCLXL, this points to a dependency of CSCs on BCLXL for survival. Importantly, ABT-737- or WEHI-539induced loss of clonogenicity could be restored when BCLXL was ectopically overexpressed (Figure 4b). To confirm that CSCs are dependent on BCLXL and not on BCL2, western blot analysis were performed for BCL2, BCLXL and BCLW proteins and indeed all spheroid cultures expressed BCLXL and small amounts of BCLW, but no BCL2 protein levels could be detected (Figure 4c). Next, we evaluated whether decreased mitochondrial priming was responsible for chemotherapy resistance of CSCs. We therefore set out to perturb the apoptotic balance with sub-lethal amounts of ABT-737, ABT-199 or WEHI-539 to determine whether this would be a means to sensitize colon CSCs toward chemotherapy. As expected, colon CSCs were resistant to single treatment with low levels of $\mathrm{BH} 3$ mimetics and also against oxaliplatin. However, when spheroid cultures were treated with ABT-737 or WEHI-539 compounds, CSCs were effectively sensitized toward oxaliplatin (Figure 4d) and other chemotherapeutic agents (Supplementary Figure 4). Similar to our observations on clonogenicity, the BCL2-specific inhibitor ABT-199 did not sensitize CSCs toward chemotherapy (Figure 4d). Finally, to determine whether combination treatment affected clonogenicity of CSCs, we used low doses of oxaliplatin that slightly reduced CSC clonogenicity (Figure 4e). However, combination of oxaliplatin with sub-lethal doses of ABT-737, but not ABT-199, resulted in further loss of clonogenicity of CSCs (Figure 4e). In agreement, when spheroid cultures were treated with ABT-737, ABT-199 or WEHI-539 in combination with oxaliplatin and growth of the cultures was measured over time (Figure 4f), we observed a strong initial decrease in cell viability in the first 4 days. However, spheroid cultures treated with oxaliplatin alone or in combination with ABT-199 regained proliferative potential in the days after, whereas combination treatment with ABT-737 or WEHI-539 strongly inhibited this revival (Figure 4f). This indicates that decreased mitochondrial priming of CSCs is an important aspect of CSCs that directs enhanced chemotherapy resistance of these cells and should thus be targeted to optimize chemotherapy. 


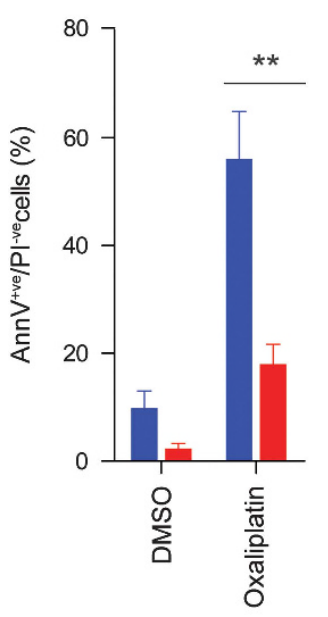

c

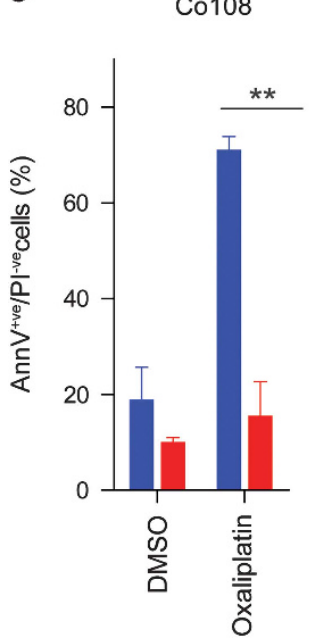

b
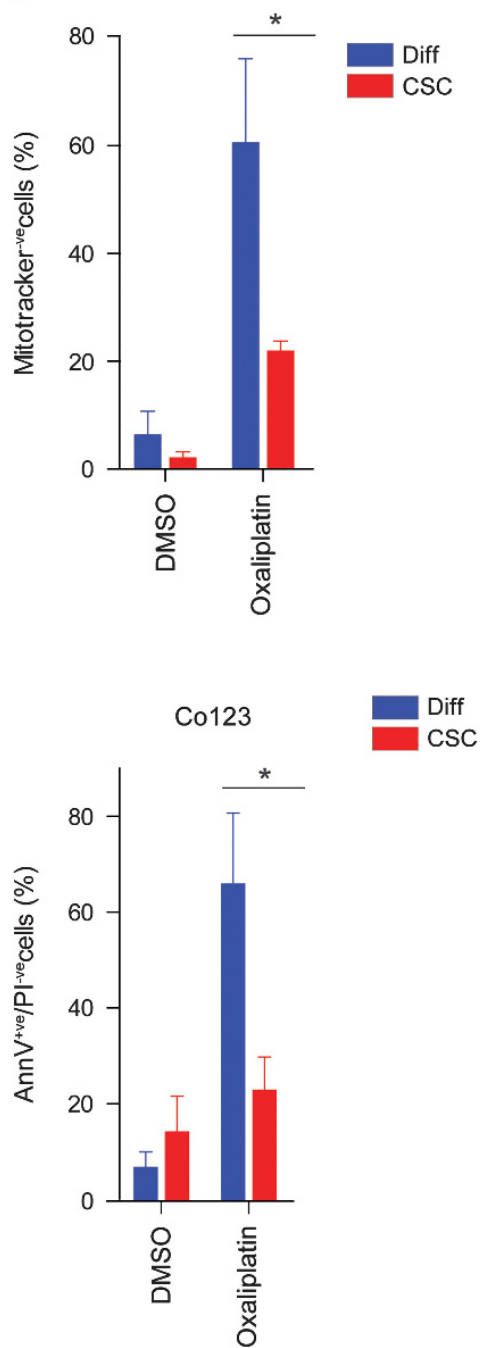

Figure 2 Colon cancer stem cell resistance can be observed with multiple techniques in various patient-derived spheroid cultures. (a) Spheroid cultures were treated with oxaliplatin for $24 \mathrm{~h}$ and (a) phosphatidylserine (PS) exposure or (b) loss of mitochondrial activity was measured. (c) Different spheroid cultures derived from different patients (C0108 and C0123) were treated with oxaliplatin and PS exposure was measured in differentiated tumor cells (TOP-GFPlow) and CSCs (TOP-GFPhigh). All spheroid cultures showed cell death in the differentiated tumor cells and not in the CSCs. Significance comparing differentiated versus CSCs is indicated $\left({ }^{\star} P<0.05,{ }^{* *} P<0.01\right)$

\section{Discussion}

In the last decade, it has become increasingly clear that many tumors are heterogeneous, consisting of CSCs and more differentiated tumor cells. Like other CSCs, colon CSCs are highly tumorigenic and therefore targeting these cells is suggested to enhance the success of cancer therapy. However, colon CSCs are resistant to chemotherapy and the molecular mechanisms that orchestrate this are still poorly understood. Here we demonstrate that colon CSCs are less mitochondrial primed and increasing mitochondrial priming by ABT-737 sensitizes colon CSCs toward therapy.

In a non-cancerous cell, there is a balance between anti-apoptotic molecules and pro-apoptotic $\mathrm{BH} 3$ molecules.

This balance determines the activation of BAX and BAK proteins and thereby mitochondrial integrity in a cell. There are two main models for activation of BAX and BAK molecules. In the first model, called the direct activation model, BH3 proteins are divided in activators (e.g. BIM and BID) and sensitizers (e.g. BAD and HRK). ${ }^{33,34}$ In this model, sensitizers can bind to anti-apoptotic molecules (e.g. BCL2 and MCL1) and can inhibit them. This inhibition of antiapoptotic molecules results in an indirect activation of BAX and BAK molecules, whereas the activators are able to activate BAX and BAK molecules directly, resulting in MOMP.

In the second model, called the neutralization model, BAX and BAK activation is a spontaneous event, which is prevented by the anti-apoptotic molecules. Anti-apoptotic $\mathrm{BCL} 2$ proteins need to be neutralized by $\mathrm{BH} 3$-only proteins to allow BAX and BAK oligomerization and MOMP. ${ }^{34,35}$ Recently, a unified model has been proposed that incorporates both models suggesting the existence of two modes of inhibition in which the BCL2 family prevents apoptosis. ${ }^{34}$

Similar to earlier studies, ${ }^{16-18,28}$ we used $\mathrm{BH} 3$ profiling to determine the level of mitochondrial priming in CSCs and differentiated cells (Figure $3 d$ ). In this assay, $\mathrm{BH} 3$ peptides were used that mimic the function of $\mathrm{BH} 3$ molecules. Using the differential specificities of the various $\mathrm{BH} 3$ molecules, it is possible to probe the mitochondrial resistance of a cell. As described before, BID and BIM are very potent in inducing mitochondrial depolarization. ${ }^{17}$ However, lower concentrations of BIM revealed lower sensitivity also toward this peptide in CSCs (Supplementary Figure 3). In both models, BIM and BID proteins can be described as potent apoptosis activators either as they represent direct inducers or because they are promiscuous in inhibiting a wide range of anti-apoptotic BCL2 members. Interestingly, CSCs were selectively more resistant to all other $\mathrm{BH} 3$ peptides that were tested. This can be due to the higher affinity of these peptides or may be explained by a loss of activators (like BID and BIM) ${ }^{28}$ in CSCs. Alternatively, it may point to the expression of higher levels of anti-apoptotic BCL2 proteins. The latter possibility is supported by the observation that simple overexpression of BCLXL, BCL2 or $B C L W$ is sufficient to prevent chemotherapy-induced apoptosis in the differentiated tumor cells, whereas the $\mathrm{BH} 3$ mimetic ABT-737 and WEHI-539 sensitizes both CSCs and the more differentiated cells. Further work is needed to determine the mechanism behind the decreased mitochondrial priming, but our findings strongly argue for a BCLXLdependent anti-apoptotic threshold in colon CSCs.

In various tumors, it has been shown that the apoptotic balance is disturbed in favor of anti-apoptotic molecules. In many lymphomas, BCL2 protein expression is increased. ${ }^{36,37}$ Moreover, also in solid tumors various anti-apoptotic proteins are shown to be highly expressed. In line with this, colorectal cancers have been reported to express enhanced levels of $B C L 2$ and BCLXL and this correlates with clinical outcome of the patient. $^{38}$ Similarly, microsatellite instable colorectal cancers frequently display loss of BAX because of a frameshift mutation ${ }^{39}$ and this correlates with decreased 5-FU sensitivity. Previously, we have shown that colon CSCs express IL-4 and this can signal in an autocrine manner. In line with our current findings, we showed that treatment of colon CSCs with neutralizing antibody against IL-4, decreased 

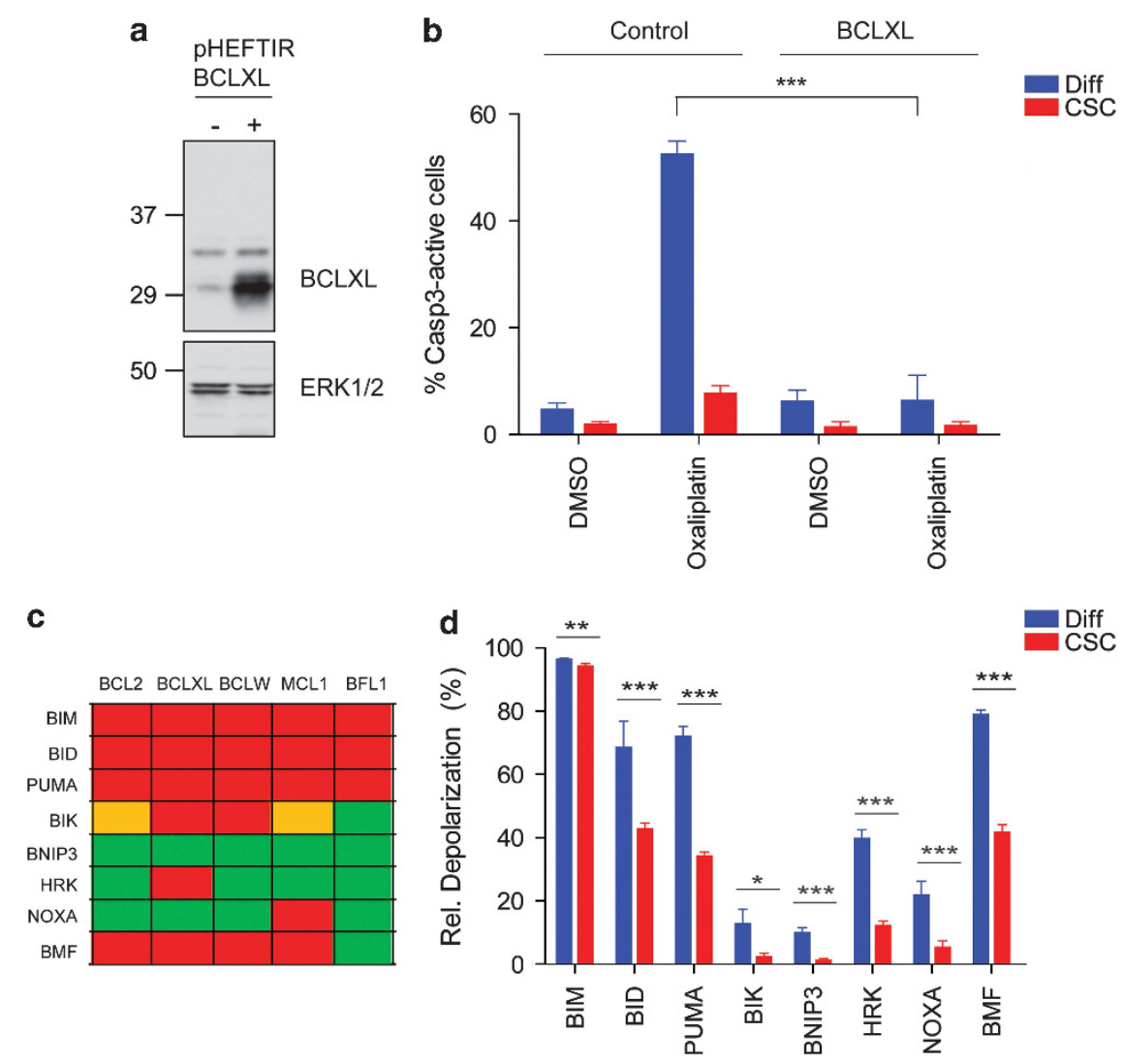

Figure 3 Colon CSCs and differentiated cells are differentially mitochondrial primed. (a) Spheroid culture (Co100) ectopically overexpressing BCLXL protein was generated, as shown by western blot analysis of BCLXL (upper panel). Lower panel shows a control western blot for ERK1/2. (b) After oxaliplatin treatment, differentiated tumor cells (TOP-GFPlow) ectopically overexpressing BCLXL are no longer sensitive and show significantly reduced cell death by caspase-3 measurement. (c) Figure showing specificity of BH3 peptides for binding to anti-apoptotic BCL2 family protein members. Red indicates high affinity and green indicates low affinity. (d) Spheroid culture (Co100) was subjected to $\mathrm{BH} 3$ profiling using various $\mathrm{BH} 3$ peptides. Cells were stained for $\mathrm{CD} 133$, treated with indicated $\mathrm{BH} 3$ peptides and mitochondrial depolarization was measured with JC1. Mitochondrial depolarization in differentiated tumor cells $\left(C D 133^{\text {low }}\right)$ and CSCs (CD133 high) is shown relative to complete depolarization obtained with CCCP. Significance comparing oxaliplatin-treated control versus BCLXL overexpressing differentiated tumor cells is indicated in $\mathbf{b}$ and comparing differentiated versus CSCs in $\mathbf{d}$ $\left({ }^{\star} P<0.05,{ }^{* \star} P<0.01,{ }^{* \star *} P<0.001\right)$

protein levels of the anti-apoptotic proteins C-FLIP, BCLXL and PED and sensitized colon CSCs to oxaliplatin treatment. ${ }^{11}$

Although our data strongly argue for a role of decreased mitochondrial priming of CSCs in chemotherapy resistance, it is important to note that therapy resistance of CSCs may have other underlying reasons. For instance, Kranenburg and colleagues have shown that colon CSCs highly express BIRC6 and knockdown of BIRC6 sensitized colon CSCs toward oxaliplatin. ${ }^{27}$ Similarly, slow cell cycling and high drug transporters activity have been connected to resistance. ${ }^{40}$ In agreement, ABCB5 was shown to be expressed in CSCs and render them drug resistant. ${ }^{41}$ The latter two mechanisms are less likely to be responsible for therapy resistance in our model, as we have previously shown that cell cycle activity is not different between CSCs and differentiated tumor cells. ${ }^{42}$ In addition, CSCs were also resistant to TRAIL, which is not dependent on either cell cycle or drug efflux. Moreover, experiments performed with doxorubicin showed no difference in uptake of the drug (not shown), indicating that drug efflux is not different in colon CSCs. Here we show that chemoresistance of CSCs is instead due to differential mitochondrial priming. Previously, we have used an inducible caspase-9 to target colon CSCs. Upon activation of caspase-9, colon CSCs were killed efficiently in vitro and in vivo. ${ }^{43}$ In line with this study, this indicates that the apoptotic block in colon CSCs is upstream of MOMP.

For many years, there is an effort to target the anti-apoptotic molecules in cancer. ABT-737, and its orally bioavailable variant $A B T-263$, target $B C L 2, B C L X L$ and $B C L W,{ }^{21}$ whereas ABT-199 and WEHI-539 specifically target BCL2 and BCLXL, respectively. ${ }^{25,26,44,45}$ Here, these three inhibitors were tested in combination with chemotherapy. Interestingly, only ABT737 and WEHI-539 were effective in sensitizing colon CSCs toward chemotherapy, whereas ABT-199 failed to kill or sensitize CSCs. In agreement, BCL2 protein expression was hardly detected in the CSCs analyzed in contrast to BCLXL. Importantly, also the more differentiated tumor cells can be sensitized by $\mathrm{BH} 3$ mimetics, which indicates that a BCLXLdependent anti-apoptotic threshold is present in differentiated cancer cells as well, but that this threshold is simply elevated in the CSCs and sufficient to block chemotherapy-induced death. As relatively low doses of $\mathrm{BH} 3$ mimetics are sufficient to sensitize CSCs, it may be feasible to design combination 


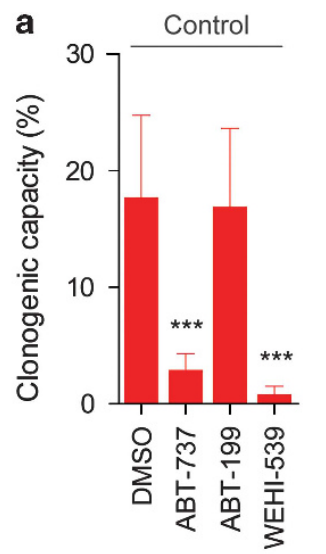

d

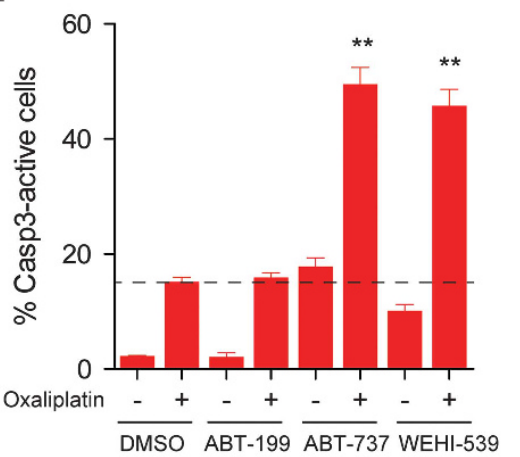

f

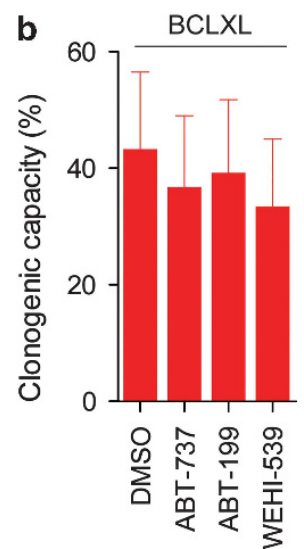

c

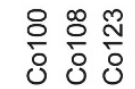

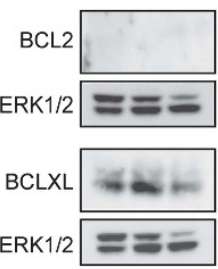

BCLW
ERK1/2 $=\ldots z$

e
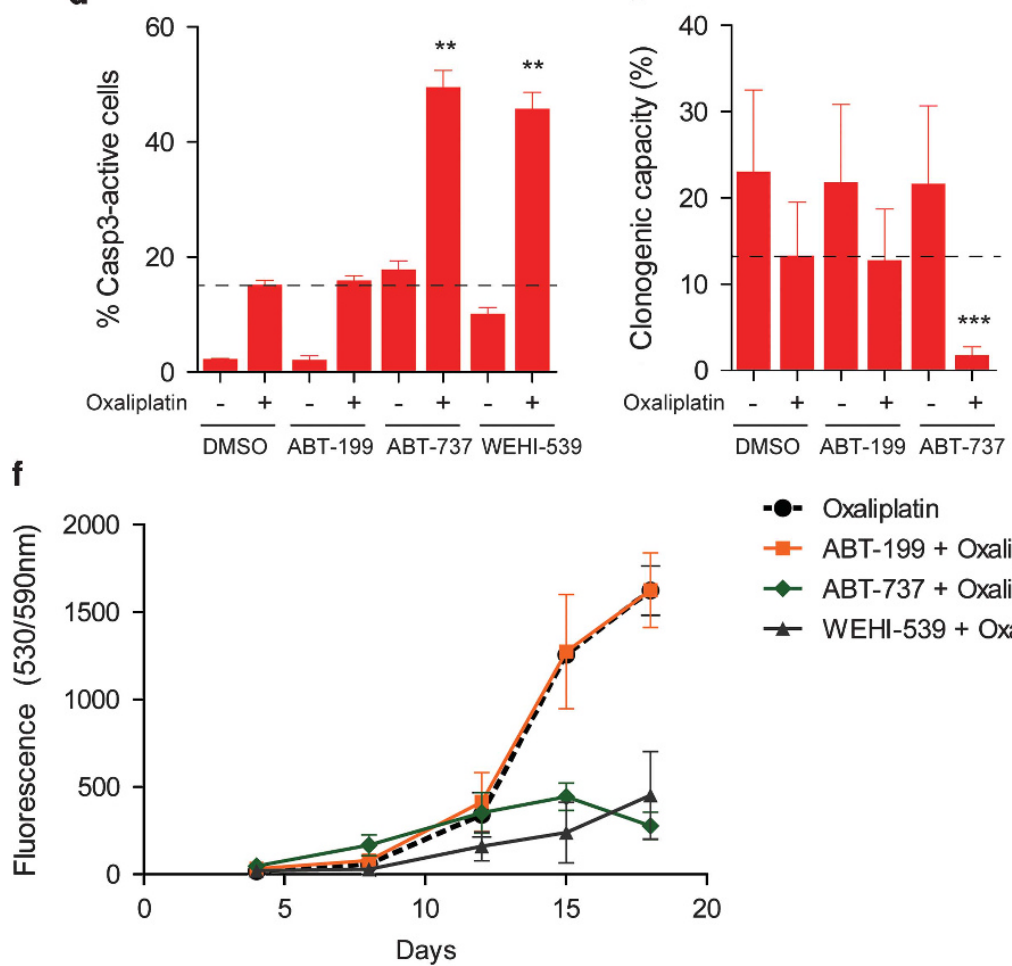

-0- Oxaliplatin

- ABT-199 + Oxaliplatin

$\leadsto$ ABT-737 + Oxaliplatin

$\neg$ WEHI-539 + Oxaliplatin

Figure 4 ABT-737 and WEHI-539 sensitize colon CSCs toward chemotherapy. (a) Untransduced or (b) BCLXL-transduced spheroid cultures were treated with $1 \mu \mathrm{M}$ of ABT-737, ABT-199 or WEHI-539 for $24 \mathrm{~h}$ and limiting dilution assay was performed on CSCs (TOP-GFPhigh). ABT-737 and WEHI-539 decreased clonogenic capacity in CSCs (a), but not when BCLXL is overexpressed (b). Significance is indicated $\left.{ }^{* \star *} P<0.001\right)$ and is related to vehicle (DMSO) alone treatment. (c) Western blot analysis on the different spheroid cultures for BCL2 (upper panel), BCLXL (middle panel) and BCLW (lower panel) proteins. Control western blots for the kinase ERK1/2, which is stably expressed in the spheroids, are shown. (d) Spheroid cultures were treated with $100 \mathrm{nM} \mathrm{ABT-737} \mathrm{or} \mathrm{WEHI-539} \mathrm{in} \mathrm{combination} \mathrm{with} 50 \mu \mathrm{M}$ oxaliplatin for $24 \mathrm{~h}$ and subsequently analyzed for caspase-3 activity or (e) clonogenic capacity using limiting dilution on CSCs (TOP-GFP ${ }^{\text {high }}$ ). Significance is indicated ( ${ }^{* *} P<0.01$ and $\left.{ }^{* * *} P<0.001\right)$ and is related to oxaliplatin alone treatment. (f) Spheroid cultures were treated with $100 \mathrm{nM} \mathrm{ABT-737,} \mathrm{ABT-199}$ or WEHI-539 in combination with $0.5 \mu \mathrm{M}$ oxaliplatin for $24 \mathrm{~h}$ and cell numbers were measured at various time points using cell titer blue

therapies that spare thrombocytes while effectively targeting colorectal cancer. Whether such combination therapies pose a threat to normal intestinal stem cells remains to be established. We have previously shown that hematopoietic stem cells are relatively unprimed, ${ }^{18}$ but whether these normal stem cells or intestinal stem cells are also sensitized by BCLXL-targeting $\mathrm{BH} 3$ mimetics remains to be determined.

Taken together, we have shown that colon CSCs are resistant to various drugs because of a decreased mitochondrial priming and that exogenous priming of CSCs with
ABT-737 or WEHI-539 is sufficient to sensitize colon CSCs toward chemotherapy.

\section{Materials and methods}

Cell culture. Colon spheroid cultures were derived from different patients with colorectal cancer in accordance with the rules of the medical ethical committee of the AMC. Briefly, primary resected human colon carcinomas were digested enzymatically for $1 \mathrm{~h}$ with collagenase $\|(1.5 \mathrm{mg} / \mathrm{ml}$; C6885 Sigma-Aldrich, Zwijndrecht, The Netherlands) at $37^{\circ} \mathrm{C}$. The dissociated samples were strained $(70 \mu \mathrm{m}$ pore size) and washed in CSC medium and subsequently cultured. Spheroid cultures were cultured in stem cell medium (advanced DMEM/F12 
(Gibco, Bleiswijk, The Netherlands)) supplemented with N-2 Supplement (Gibco), $6 \mathrm{mg} / \mathrm{ml}$ glucose, $5 \mathrm{mM}$ HEPES, $2 \mathrm{mM}$ L-glutamine, $4 \mu \mathrm{g} / \mathrm{ml}$ heparin, epidermal growth factor $(50 \mathrm{ng} / \mathrm{ml})$ and basic fibroblast growth factor $(10 \mathrm{ng} / \mathrm{ml})$. Spheroid cultures were maintained in ultra-low adherent flasks (Corning, Amsterdam, The Netherlands). The vector for the TCF/LEF reporter driving expression of GFP (TOP-GFP) was a gift from Dr. Laurie Ailles and was described previously. ${ }^{46}$ Spheroid cultures were transduced lentivirally with TCF/LEF reporter and single cell cloned by single-cell plating in 96-well ultra-low adhesion plates (Corning) with FACSaria (BD Biosciences, Breda, The Netherlands). To generate spheroid cultures that ectopically overexpress $B C L X L$, spheroid cultures were transduced with pHEFTIR-BCLXL. The lentiviral construct PHEFTIG was a kind gift from Arjen Bakker and Dr. Bianca Blom (AMC, Amsterdam, The Netherlands). First GFP within pHEFTIG was replaced by RFP to generate pHEFTIR. Next, BCLXL was cloned into PHEFTIR to generate a pHEFTIR-BCLXL. Spheroid cultures were transduced with pHEFTIR (control) or pHEFTIR-BCLXL (BCLXL). To generate spheroid culture expressing BCL2, BCLW, BCLXL, BFL1, BCL proteins, spheroid cultures were retroviral transduced with pMX-IRES-Blasticidin with the CDNA of the particular BCL2 protein and selected for at least 2 weeks. These constructs were kind gift from Professor Jannie Borst (The Netherlands Cancer Institute, Amsterdam, The Netherlands) and were described previously. ${ }^{19}$ Spheroid culture ectopically expressing MCL1-3a mutant was generated using LZRS-MCL1-3A, which was a gift from Dr. Mien-Chie Hung (The University of Texas MD Anderson Cancer Center, Houston, TX, USA). This MCL1-3A mutant construct was previously described and was shown that it cannot be degraded by the E3 ligase $\beta$-TrCP. ${ }^{47}$

Reagents. The following chemotherapeutic compounds were tested: $50 \mu \mathrm{M}$ oxaliplatin, $10 \mu \mathrm{g} / \mathrm{ml}$ etoposide, $200 \mu \mathrm{g} / \mathrm{ml} 5$-FU (all from Sigma-Aldrich), $50 \mu \mathrm{M}$ cisplatin (Platosin, Pharmachemie, Haarlem, The Netherlands) all for $24 \mathrm{~h}$, and $100 \mathrm{ng} / \mathrm{ml}$ rhTRAIL (Enzo Life Sciences, Raamsdonkveer, The Netherlands) in combination with $1.5 \mu \mathrm{g} / \mathrm{ml}$ anti-FLAG antibody (Sigma-Aldrich) for $4 \mathrm{~h}$. FOLFOX and FOLFIRI treatments consisted of $1.25 \mu \mathrm{M}$ oxaliplatin (FOLFOX) or $1 \mu \mathrm{M}$ irinotecan (Sigma-Aldrich) treatment followed after $90 \mathrm{~min}$ by $50 \mu \mathrm{M} \mathrm{5-FU,} \mathrm{cells}$ were analyzed after $24 \mathrm{~h}$. Spheroid cultures were treated for $24 \mathrm{~h}$ with ABT-199, ABT-737 (Selleck Chemicals, Huissen, The Netherlands) or WEHI-539 (ChemScene, Monmouth Junction, NJ, USA) in combination with a chemotherapeutic compound or only vehicle.

Immunoblotting and antibodies. Cells were lysed in $1 \times$ RIPA lysis and extraction buffer (Thermo Fisher Scientific, Etten-Leur, The Netherlands) containing complete protease inhibitor (Roche, Woerden, The Netherlands). After clearing the lysate by high-speed centrifugation (14000 r.p.m., $10 \mathrm{~min}, 4^{\circ} \mathrm{C}$ ), the protein concentration was determined using BCA protein assay (Thermo Scientific, Breda, The Netherlands). $20 \mu \mathrm{g}$ extracted proteins was separated by $12 \%$ precast gels (Bio-Rad, Veenendaal, The Netherlands) and transferred to Hybond-P membranes (Amersham, Upsalla, Sweden). Membranes were blocked with 5\% milk in phosphate-buffered saline solution containing $0.2 \%$ TWEEN (PBS-T) for $1 \mathrm{~h}$. The membranes were incubated with the appropriate amount of antibody. Primary antibody incubations were carried out in $2.5 \%$ milk/PBS-T overnight at $4{ }^{\circ} \mathrm{C}$ and subsequently washed three times with PBS-T. Membranes were then incubated with anti-mouse IgG horseradish peroxidase conjugates for $1 \mathrm{~h}$, washed three times and detection of bound antibody was performed with ECLplus reagents (Amersham). Western blots were analyzed by LAS4000. The antibodies used were anti-BCLXL (clone S18, 1:500, Santa-Cruz, Heidelberg, Germany), anti-BCL2 (clone N19, 1:500, Santa-Cruz), anti-BCLW (clone 31H4, 1:500, Abcam, Cambridge, UK), anti-BFL1 (1:250, Santa-Cruz), anti-MCL1 (clone 4572, 1:1000, Cell Signaling, Leiden, The Netherlands) and anti-ERK1/2 (1:10000, Cell Signaling).

RNA extraction, cDNA synthesis and RT-PCR. RNA was extracted from cells using Trizol reagent (Invitrogen, Leek, The Netherlands) in accordance with the manufacturer's protocol. RNA concentration was determined with NanoDrop ND-2000 (Thermo Scientific) and $1 \mu \mathrm{g}$ of RNA was used to synthesis CDNA using SuperScript III in accordance with the manufacturer's protocol (Invitrogen). RT-PCR was performed with LC480 SYBR green (Roche) in accordance with the manufacturer's instructions on a LC480. The following primers were used: $18 \mathrm{~S}$ sense: $5^{\prime}$-AGACAACAAGCTCCGTGAAGA-3', 18S antisense: 5'-CAGAAGTGACGCAGCCCTCTA-3', BCLB sense: 5'-CTAAGGAGCAGGAG GGCG-3', and BCLB anti-sense: 5'-GTGGAAAGGGGGTCCTGAAG-3'.
Limiting-dilution assay. Spheroid cultures were dissociated and the $10 \%$ TOP-GFP high and 10\% TOP-GFPlow cells were FACS deposited using FACSaria (BD Biosciences) in a limiting dilution manner at 1, 2, 4, 8, 16, 32, 64, 128 and 256 cells per well in ultra-low 96 -well plates (Corning). Clonal frequency was evaluated with the Extreme Limiting Dilution Analysis 'limdil' function as described. ${ }^{48}$

FACS staining. Various colon spheroid cultures transduced with TOP-GFP were dissociated and were stained with AC133/CD133-APC antibody $(1: 25$, Miltenyi Biotec, Paris, France) in PBS containing $1 \%$ bovine serum albumin (PBS-B) for $30 \mathrm{~min}$ at $4{ }^{\circ} \mathrm{C}$. Subsequently, cells were washed with PBS-B and resuspended in PBS-B. 7-AAD (BD Biosciences) was used to exclude death cells. Staining was analyzed on a FASCanto (BD Biosciences).

Cell death assays. Primary colon cancer spheroid cultures were dissociated with trypsin-EDTA and seeded as single cells on an adherent cell culture 12-well plate (Greiner, Alphen a/d Rijn, The Netherlands) overnight. The next day, adherent cells were treated with chemotherapeutic drugs or targeted compounds for the indicated times. After treatment, cells were harvested and identification of the CSCs was performed by gating on the TOP-GFPhigh cells in the spheroid cultures, whereas the differentiated cells were identified in the same cultures by gating on the TOP-GFPlow cells. To measure apoptosis at the single-cell level in both populations, three distinct FACS-based measurements of cell death were used. The first, caspase 3 activity was measured with CaspGlow active staining kit (Red-DEVD-FMK) according to the manufacturer's instructions (BioVision, Milpitas, CA, USA). In short, after treatment, spheroid cultures were made single cells using trypsin-EDTA. 50000 cells were washed with stem cell medium and stained with RED-DEVD-FMK for $1 \mathrm{~h}$ at $37^{\circ} \mathrm{C}$. Subsequently, cells were washed twice with wash buffer. The second FACS-based measurement of cell death was based on the exposure of phospatidylserine. Spheroid cultured cells were stained with Annexin V-APC (BD biosciences) and 7-AAD (BD biosciences) for $15 \mathrm{~min}$ at RT. The third assay involved mitochondrial activity, which was measured with Mitotracker Orange CMTMRos (Molecular Probes, Bleiswijk, The Netherlands). Spheroid cultures were incubated for $30 \mathrm{~min}$ with $25 \mathrm{nM}$ Mitotracker Orange at $37^{\circ} \mathrm{C}$. All three apoptosis staining methods were followed by flow cytometry performed with FACSCanto (BD biosciences). Cell death was measured in CSCs by gating on TOP-GFP high cells and in differentiated tumor cells by gating on TOP-GFPlow cells.

Cell survival assay. Primary colon cancer spheroid cultures were dissociated and seeded as in cell death assays. Adherent cells were treated with oxaliplatin in combination with ABT-737, ABT-199 or WEHI-539. After $24 \mathrm{~h}$ treatment, cells were harvested and 2000 cells were transferred into ultra-low adherent 96-well plates (Corning). Cell survival was measured at different time points by adding $20 \mu /$ well of cell titer blue reagent (Promega, Leiden, The Netherlands). Subsequently, cells were incubated for $4 \mathrm{~h}$ and fluorescence was measured on a Biotek HT synergy plate reader (BioTek, Potton, UK).

BH3 profiling. Mitochondrial priming was measured as described before. ${ }^{16}$ Briefly, spheroid cultures were stained with AC133/CD133-APC antibody (1:25, Miltenyi Biotec). Subsequently, cells were washed in T-EB (300 mM Trehalose, $10 \mathrm{mM}$ HEPES-KOH pH 7.7, $80 \mathrm{mM} \mathrm{KCl}, 1 \mathrm{mM}$ EGTA, $1 \mathrm{mM}$ EDTA, 0.1\% BSA, $5 \mathrm{mM}$ succinate) and 50000 cells resuspended in $100 \mu \mathrm{l} \mathrm{T-EB}$ were added to $100 \mu \mathrm{l}$ of T-EB containing $20 \mu \mathrm{g} / \mathrm{ml}$ digitonin, $20 \mu \mathrm{g} / \mathrm{ml}$ oligomycin and peptides. Cells were incubated for $90 \mathrm{~min}$ with various $100 \mu \mathrm{M} \mathrm{BH} 3$ peptides at RT. As shown before, BIM and BID peptides were very potent and therefore 6 and $12 \mu \mathrm{m}$ of these peptides were tested, respectively. More concentrations of BIM peptides are tested and shown in Supplementary Figure 4. JC-1 staining was performed for $30 \mathrm{~min}$ at RT $(1 \mu \mathrm{M})$ and MOMP was measured in CSCs (10\% CD133 ${ }^{\text {high }}$ cells) and more differentiated cells $\left(10 \%\right.$ CD $133^{\text {low }}$ cells) on a FACSCanto (BD Bioscience).

The following BH3 peptides (Eurogentec, Maastricht, The Netherlands) were used: BIM: MRPEIWIAQELRRIGDEFNA;

BID: EDIIRNIARHLAQVGDSMDR;

PUMA: EQWAREIGAQLRRMADDLNA;

BIK: MEGSDALALRLACIGDEMDV;

BNIP3: VVEGEKEVEALKKSADWVSD;

HRK: SSAAQLTAARLKALGDELHQ;

NOXA A: AELPPEFAAQLRKIGDKVYC;

BMF: HQAEVQIARKLQLIADQFHR 


\section{Conflict of Interest}

The authors declare no conflict of interest.

Acknowledgements. We thank Louis Vermeulen, Felipe de Sousa e Melo, Michael Bots, Maarten Bijlsma and Jeremy Ryan for constructive discussion, and the surgeons Professor Bemelman, Professor Gulik, Dr. Tanis, Dr. Buskens and Dr. Van de Ven (AMC, Amsterdam) for providing colon cancer samples. We also thank Dr. Mien-Chie Hung (University of Texas) for providing MCL1-3a mutant construct and Dr. Rogier Rooswinkel and Professor Jannie Borst (The Netherlands Cancer Institute, Amsterdam) for providing all other pro-survival BCL2 family members, Dr. Laurie Ailles (University of Toronto) for providing Tcf/Lef reporter construct and Arjen Bakker and Dr. Bianca Blom (AMC Amsterdam) for providing pHEFTIG construct. Finally, we thank Berend Hooibrink, Toni van Capel and Kate Cameron (AMC, Amsterdam) for assistance with fluorescence-activated cell sorting experiments. This work was supported by a $\mathrm{VICl}$ grant from the Netherlands Organisation for Scientific Research and a Dutch Cancer Society (KWF Kankerbestrijding) grant (2009-4416 and 2012-5612; to JPM).

1. Jemal A, Bray F, Center MM, Ferlay J, Ward E, Forman D. Global cancer statistics. CA Cancer J Clin 2011; 61: 69-90.

2. Ferlay J, Shin HR, Bray F, Forman D, Mathers C, Parkin DM. Estimates of worldwide burden of cancer in 2008: GLOBOCAN 2008. Int J Cancer 2010; 127: 2893-2917.

3. Van Cutsem E, Nordlinger B, Cervantes A. Group EGW. Advanced colorectal cancer: ESMO Clinical Practice Guidelines for treatment. Ann Oncol 2010; 21(Suppl 5): v93-v97.

4. Cunningham D, Humblet $Y$, Siena $S$, Khayat D, Bleiberg $H$, Santoro A et al. Cetuximab monotherapy and cetuximab plus irinotecan in irinotecan-refractory metastatic colorectal cancer. New Engl J Med 2004; 351: 337-345.

5. Hurwitz H, Fehrenbacher L, Novotny W, Cartwright T, Hainsworth J, Heim W et al. Bevacizumab plus irinotecan, fluorouracil, and leucovorin for metastatic colorectal cancer. New Engl J Med 2004; 350: 2335-2342.

6. Punt CJ, Tol J. More is less-combining targeted therapies in metastatic colorectal cancer Nat Rev Clin Oncol 2009; 6: 731-733.

7. Kemper K, Grandela C, Medema JP. Molecular identification and targeting of colorectal cancer stem cells. Oncotarget 2010; 1: 387-395.

8. Todaro M, Francipane MG, Medema JP, Stassi G. Colon cancer stem cells: promise of targeted therapy. Gastroenterology 2010; 138: 2151-2162.

9. Ricci-Vitiani L, Lombardi DG, Pilozzi E, Biffoni M, Todaro M, Peschle C et al. Identification and expansion of human colon-cancer-initiating cells. Nature 2007; 445: 111-115.

10. O'Brien CA, Pollett A, Gallinger S, Dick JE. A human colon cancer cell capable of initiating tumour growth in immunodeficient mice. Nature 2007; 445: 106-110.

11. Todaro M, Alea MP, Di Stefano AB, Cammareri $P$, Vermeulen $L$, lovino $F$ et al. Colon cancer stem cells dictate tumor growth and resist cell death by production of interleukin-4. Cell Stem Cell 2007; 1: 389-402.

12. Vermeulen L, De Sousa EMF, van der Heijden M, Cameron K, de Jong JH, Borovski T et al. Wnt activity defines colon cancer stem cells and is regulated by the microenvironment. Nat Cell Biol 2010; 12: 468-476.

13. Medema JP. Cancer stem cells: the challenges ahead. Nat Cell Biol 2013; 15: 338-344.

14. Dylla SJ, Beviglia L, Park IK, Chartier C, Raval J, Ngan L et al. Colorectal cancer stem cells are enriched in xenogeneic tumors following chemotherapy. PLoS One 2008; 3: e2428.

15. de Bruin EC, Medema JP. Apoptosis and non-apoptotic deaths in cancer development and treatment response. Cancer Treat Rev 2008; 34: 737-749.

16. Ryan JA, Brunelle JK, Letai A. Heightened mitochondrial priming is the basis for apoptotic hypersensitivity of CD4 + CD8 + thymocytes. Proc Natl Acad Sci USA 2010; 107 12895-12900.

17. Ni Chonghaile T, Sarosiek KA, Vo TT, Ryan JA, Tammareddi A, Moore Vdel G et al Pretreatment mitochondrial priming correlates with clinical response to cytotoxic chemotherapy. Science 2011; 334: 1129-1133.

18. Vo TT, Ryan J, Carrasco R, Neuberg D, Rossi DJ, Stone RM et al. Relative mitochondrial priming of myeloblasts and normal HSCs determines chemotherapeutic success in AML. Cell 2012; 151: 344-355.

19. Rooswinkel RW, van de Kooij B, Verheij M, Borst J. Bcl-2 is a better ABT-737 target than $\mathrm{Bcl}-\mathrm{xL}$ or Bcl-w and only Noxa overcomes resistance mediated by Mcl-1, Bfl-1, or Bcl-B. Cell Death Dis 2012; 3: e366.

20. Zhai D, Jin C, Satterthwait AC, Reed JC. Comparison of chemical inhibitors of antiapoptotic Bcl-2-family proteins. Cell Death Differ 2006; 13: 1419-1421.

21. Oltersdorf T, Elmore SW, Shoemaker AR, Armstrong RC, Augeri DJ, Belli BA et al. An inhibitor of Bcl-2 family proteins induces regression of solid tumours. Nature 2005; 435 : 677-681.
22. Merino D, Khaw SL, Glaser SP, Anderson DJ, Belmont LD, Wong $C$ et al. Bcl-2, Bcl-x $(\mathrm{L})$, and $\mathrm{Bcl}-\mathrm{w}$ are not equivalent targets of ABT-737 and navitoclax (ABT-263) in lymphoid and leukemic cells. Blood 2012; 119: 5807-5816.

23. Wilson WH, O'Connor OA, Czuczman MS, LaCasce AS, Gerecitano JF, Leonard JP et al. Navitoclax, a targeted high-affinity inhibitor of BCL-2, in lymphoid malignancies: a phase 1 dose-escalation study of safety, pharmacokinetics, pharmacodynamics, and antitumour activity. Lancet Oncol 2010; 11: 1149-1159.

24. Gandhi L, Camidge DR, Ribeiro de Oliveira M, Bonomi P, Gandara D, Khaira D et al. Phase I study of Navitoclax (ABT-263), a novel Bcl-2 family inhibitor, in patients with small-cell lung cancer and other solid tumors. J Clin Oncol 2011; 29: 909-916.

25. Souers AJ, Leverson JD, Boghaert ER, Ackler SL, Catron ND, Chen J et al. ABT-199, a potent and selective BCL-2 inhibitor, achieves antitumor activity while sparing platelets. Nat Med 2013; 19: 202-208.

26. Lessene G, Czabotar PE, Sleebs BE, Zobel K, Lowes KN, Adams JM et al. Structure-guided design of a selective BCL-X(L) inhibitor. Nat Chem Biol 2013; 9 : 390-397.

27. Van Houdt WJ, Emmink BL, Pham TV, Piersma SR, Verheem A, Vries RG et al. Comparative proteomics of colon cancer stem cells and differentiated tumor cells identifies BIRC6 as a potential therapeutic target. Mol Cell Proteomics 2011; 10: M111 011353.

28. Deng J, Carlson N, Takeyama K, Dal Cin P, Shipp M, Letai A. BH3 profiling identifies three distinct classes of apoptotic blocks to predict response to ABT-737 and conventional chemotherapeutic agents. Cancer Cell 2007; 12: 171-185.

29. Certo M, Del Gaizo Moore V, Nishino M, Wei G, Korsmeyer S, Armstrong SA et al. Mitochondria primed by death signals determine cellular addiction to antiapoptotic BCL-2 family members. Cancer Cell 2006; 9: 351-365.

30. Pegoraro L, Palumbo A, Erikson J, Falda M, Giovanazzo B, Emanuel BS et al. A 14;18 and an 8;14 chromosome translocation in a cell line derived from an acute B-cell leukemia. Proc Natl Acad Sci USA 1984; 81: 7166-7170.

31. Sinicrope FA, Ruan SB, Cleary KR, Stephens LC, Lee JJ, Levin B. Bcl-2 and p53 oncoprotein expression during colorectal tumorigenesis. Cancer Res 1995; 55: 237-241.

32. Ben-Ezra JM, Kornstein MJ, Grimes MM, Krystal G. Small cell carcinomas of the lung express the Bcl-2 protein. Am J Pathol 1994; 145: 1036-1040.

33. Letai A, Bassik MC, Walensky LD, Sorcinelli MD, Weiler S, Korsmeyer SJ. Distinct BH3 domains either sensitize or activate mitochondrial apoptosis, serving as prototype cancer therapeutics. Cancer Cell 2002; 2: 183-192.

34. Llambi F, Moldoveanu T, Tait SW, Bouchier-Hayes L, Temirov J, McCormick LL et al. A unified model of mammalian BCL-2 protein family interactions at the mitochondria. Mol Cell 2011; 44: 517-531.

35. Willis SN, Fletcher JI, Kaufmann T, van Delft MF, Chen L, Czabotar PE et al. Apoptosis initiated when $\mathrm{BH} 3$ ligands engage multiple Bcl-2 homologs, not Bax or Bak. Science 2007; 315: 856-859.

36. Weiss LM, Warnke RA, Sklar J, Cleary ML. Molecular analysis of the t(14;18) chromosomal translocation in malignant lymphomas. New Engl J Med 1987; 317: 1185-1189.

37. Aisenberg AC, Wilkes BM, Jacobson JO. The bcl-2 gene is rearranged in many diffuse B-cell lymphomas. Blood 1988; 71: 969-972.

38. Ogura E, Senzaki H, Yamamoto D, Yoshida R, Takada H, Hioki K et al. Prognostic significance of $\mathrm{Bcl}-2, \mathrm{Bcl}-\mathrm{xL} / \mathrm{S}, \mathrm{Bax}$ and Bak expressions in colorectal carcinomas. Oncol Rep 1999; 6: 365-369.

39. Rampino N, Yamamoto H, lonov Y, Li Y, Sawai H, Reed JC et al. Somatic frameshift mutations in the BAX gene in colon cancers of the microsatellite mutator phenotype. Science 1997; 275: 967-969.

40. Dean M, Fojo T, Bates S. Tumour stem cells and drug resistance. Nat Rev Cancer 2005; 5: 275-284

41. Wilson BJ, Schatton T, Zhan Q, Gasser M, Ma J, Saab KR et al. ABCB5 identifies a therapy-refractory tumor cell population in colorectal cancer patients. Cancer Res 2011; 71: 5307-5316.

42. Kemper K, Prasetyanti PR, De Lau W, Rodermond H, Clevers H, Medema JP. Monoclonal antibodies against Lgr5 identify human colorectal cancer stem cells. Stem Cells 2012; 30: 2378-2386.

43. Kemper K, Rodermond H, Colak S, Grandela C, Medema JP. Targeting colorectal cancer stem cells with inducible caspase-9. Apoptosis 2012; 17: 528-537.

44. Vandenberg CJ, Cory S. ABT-199, a new Bcl-2-specific BH3 mimetic, has in vivo efficacy against aggressive Myc-driven mouse lymphomas without provoking thrombocytopenia. Blood 2013; 121: 2285-2288.

45. Davids MS, Letai A. ABT-199: taking dead aim at BCL-2. Cancer Cell 2013; 23: 139-141.

46. Reya T, Duncan AW, Ailles L, Domen J, Scherer DC, Willert $K$ et al. A role for Wnt signalling in self-renewal of haematopoietic stem cells. Nature 2003; 423: 409-414.

47. Ding Q, He X, Hsu JM, Xia W, Chen CT, Li LY et al. Degradation of MCl-1 by beta-TrCP mediates glycogen synthase kinase 3-induced tumor suppression and chemosensitization. Mol Cell Biol 2007; 27: 4006-4017.

48. Hu Y, Smyth GK. ELDA: extreme limiting dilution analysis for comparing depleted and enriched populations in stem cell and other assays. J Immunol Methods 2009; 347: 70-78. 